\title{
DigesTower: serious game como estratégia para prevenção e enfrentamento da obesidade infantil
}

\author{
Jéssica D. Dias ${ }^{1}$, Marcelo S. Mekaro ${ }^{2}$, Jennifer K. C. Lu ${ }^{3}$, Gabriel S. Sorrentino ${ }^{3}$ \\ Marcos Tsuda ${ }^{3}$, Joice L. Otsuka ${ }^{4}$, Delano M. Beder ${ }^{4}$ \\ Silvia H. Zem-Mascarenhas².
}

${ }^{1}$ Escola de Enfermagem de Ribeirão Preto (EERP) - Universidade de São Paulo (USP) - Ribeirão Preto - SP - Brazil

${ }^{2}$ Departmento de Enfermagem (DEnf) - Universidade Federal de São Carlos (UFSCar) - São Carlos - SP - Brazil

${ }^{3}$ Laboratório de Objetos de Aprendizagem (LOA) - Universidade Federal de São Carlos (UFSCar) - São Carlos - SP - Brazil

${ }^{3}$ Departamento de Computação (DC) - Universidade Federal de São Carlos (UFSCar) São Carlos - SP - Brazil

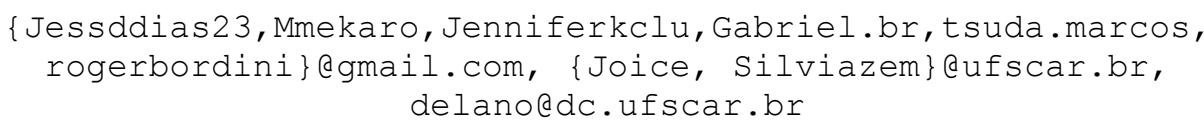

Resumo. Este estudo tem como objetivo descrever um serious game desenvolvido como uma estratégia a fim de auxiliar programas de enfrentamento da obesidade infantil. $O$ desenvolvimento do jogo percorreu até o momento as seguintes etapas: conceito, pré-produção, prototipação, produção de uma versão jogável (alpha) e avaliação do mesmo por especialistas em saúde e computação. O jogo DigesTower se passa dentro do sistema digestivo humano e se destaca por unir a temática de alimentação saudável à mecânica de jogos Tower Defense, com vistas a estimular o aprendizado do jogador e incentivar mudanças de comportamento.

\section{Cenário de uso}

A obesidade se tornou um grande desafio para a saúde pública, uma vez que sua incidência e prevalência cresceram de forma preocupante nos últimos anos [Reis et al 2011]. A fim de resolver esta problemática, as políticas de saúde têm estabelecido um conjunto de metas interdisciplinares visando à promoção a saúde e combate à obesidade [Baptista and Lima 2006, Brasil 2015].

Ainda, as crianças atuais são consideradas nativas digitais, ou seja, a geração que nasceu na era tecnológica e estão acostumadas desde a infância a navegar na internet e com uma linguagem interativa, dificultando a adaptação a um sistema de ensino tradicional [Savi and Ulbricht 2008]. Percebe-se a necessidade de atividades de educação em saúde e o uso de estratégias diferenciadas para serem aplicadas a esse público infantil, como é o caso dos jogos digitais [Toscani et al 2007].

Os serious games (jogo sérios) podem ser ferramentas úteis e de boa receptividade por parte de crianças para atingir o objetivo da educação em saúde. O uso 
de tecnologias pode oferecer benefícios significativos para o processo educativo, favorecendo a aprendizagem e motivando a criança a aderir ao tratamento.

Assim, surge à importância da inserção de um serious game para a promoção de uma alimentação e hábitos mais saudáveis. Considerando a utilização de tecnologias por crianças, acredita-se na potencialidade desses recursos para a mudança de comportamentos nessa população, particularmente com relação à obesidade. Este estudo tem como objetivo descrever um serious game desenvolvido como uma estratégia a fim de auxiliar as estratégias de enfrentamento da obesidade infantil.

Diante desse cenário, a proposta deste serious game é fazer a criança compreender melhor a digestão dentro do corpo humano, bem como diferenciar os tipos de alimentos, entender quais deles são mais saudáveis e suas composições. O DigesTower pode ser jogado por crianças em idade escolar, ou em geral, por crianças que já consigam ler e usar um computador ou dispositivo móvel como um tablet. O jogo pode ser utilizado como uma estratégia educativa adicional de combate à obesidade infantil, especialmente em programas interdisciplinares de prevenção e tratamento da doença.

\section{Desenvolvimento}

O desenvolvimento do serious game foi realizado em um período de um ano por quatro estudantes de graduação (Ciência da computação, Enfermagem, Imagem e Som e Música) e uma aluna de mestrado em Enfermagem, sob a coordenação de docentes das áreas de Computação e Enfermagem.

Por se tratar de um grupo interdisciplinar, o jogo foi desenvolvido segundo uma abordagem colaborativa e equilibrada: enquanto os programadores e os ilustradores se empenham na elaboração de uma mecânica divertida e funcional ao jogador, os docentes e alunos responsáveis pelo conteúdo educacional construíram um sistema de aprendizagem significativo e didático, de forma que não fosse ofuscada pela narrativa ou outros elementos, e foi considerado o maior desafio do projeto.

O jogo foi idealizado para funcionamento multiplataforma, para ser utilizado em computadores e Tablets e desenvolvido em formato 2D, utilizando a engine Unity $3 D$. Essa engine foi escolhida devido a flexibilidade em se trabalhar com jogos 3D e 2D bem como a portabilidade entre as diferentes plataformas Android, iOS, Web, entre outras. Foi utilizada também JavaScript, uma linguagem interpretada que normalmente é usada para executar scripts do lado do usuário apenas, sem necessidade de comunicação com o servidor. Devido ao fato de ser suportado por quase a totalidade de navegadores, é uma boa opção para atingir uma grande quantidade de jogadores.

Os estudos de Novak (2011) e Schell (2011) foram escolhidos para o delineamento metodológico do jogo e auxílio à construção dos documentos de design necessários. O roteiro utilizado para elaboração dos documentos foi desenvolvido juntamente a uma equipe interdisciplinar.

O desenvolvimento do serious game percorreu até o momento as seguintes etapas: conceito, pré-produção (documentação do jogo), prototipação, produção de um jogo educacional em versão alpha e avaliação de especialistas em saúde e computação.

O jogo já passou por uma avaliação de sua primeira versão jogável, utilizando questionários semiestruturados com base no método de avaliação de jogos educacionais 
EGameFlow [Fu Su and $\mathrm{Yu}$ 2009]. O EGameFlow é uma adaptação para jogos educacionais derivada do método GameFlow, que tem como foco a avaliação de jogos digitais com vistas ao seu potencial de fornecer ao jogador uma experiência de diversão e prazer [Sweetser and Wyeth, 2005].

O instrumento de coleta de dados utilizado para a avaliação dos especialistas da área da saúde com base no método EGameFlow foi inicialmente adaptado, validado e aplicado previamente para avaliações de outros jogos educacionais pelo Laboratório de Objetos de Aprendizagem (LOA) [Tsuda et al 2014] e foi solicitada autorização aos autores para uso do mesmo neste estudo em questão.

\section{Apresentação do Software}

O DigesTower foi produzido até sua versão alpha na qual suas propriedades e funcionalidades já foram implementadas. No entanto, esta versão ainda contém alguns erros, tais como problemas de balanceamento e interface [Benthke 2003].

A versão alpha do DigesTower apresenta três fases (constituídas de sete níveis gradativos de dificuldade). O jogo se passa dentro do sistema digestivo da personagem principal (Elise) e é considerado um jogo do tipo tower defense. Jogos Tower Defense podem ser classificados como um subtipo dos jogos de estratégia, e como seu nome sugere, com enfoque na defesa de um elemento do jogo [Zechner and Green 2012].

No DigesTower, a Elise é uma criança que está com fome e vai até a geladeira escolher o que comer, assim, o jogo se inicia. Os alimentos são os "inimigos"1 e as enzimas digestivas são as torres de defesa. Ao iniciar o jogo há uma breve explicação do que são os alimentos, bem como ilustrações dos mesmos e a classe que os representa (carboidratos, proteínas e gorduras). Ao longo do jogo é ilustrado o sistema digestivo humano e seus principais órgãos. Também há momentos de explicações sobre a digestão de cada classe alimentar. Ao final de cada fase há uma conclusão geral sobre os alimentos e com relação à saúde da personagem. Esta versão encontra-se disponível para acesso no website do LOA $^{2}$.

O jogo desenvolvido apresenta uma licença do tipo Creative Commons, pois se trata de um recurso educacional aberto voltado para o uso e reuso da comunidade. Os pacotes de instalação do jogo, bem como os códigos fontes, serão compartilhados com a comunidade de software livre. Tal recurso educacional, bem como todos seus componentes, será disponibilizado como um Recurso Educacional Aberto (REA) no Repositório Digital Livre Saber, de acesso livre da SEaD/UFSCar, no qual há um vasto acervo de objetos educacionais multimídia produzidos como recursos educacionais abertos. A seguir são apresentadas algumas telas desta primeira versão do jogo DigesTower.

\footnotetext{
1 "Inimigos", em jogos do estilo tower defense, são os elementos que vêm em ondas e precisam ser destruídos pelas torres de defesa. No DigesTower os alimentos vêm em ondas e precisam ser digeridos pelas enzimas digestivas.

${ }^{2}$ http://www.loa.sead.ufscar.br/digestower.php
} 


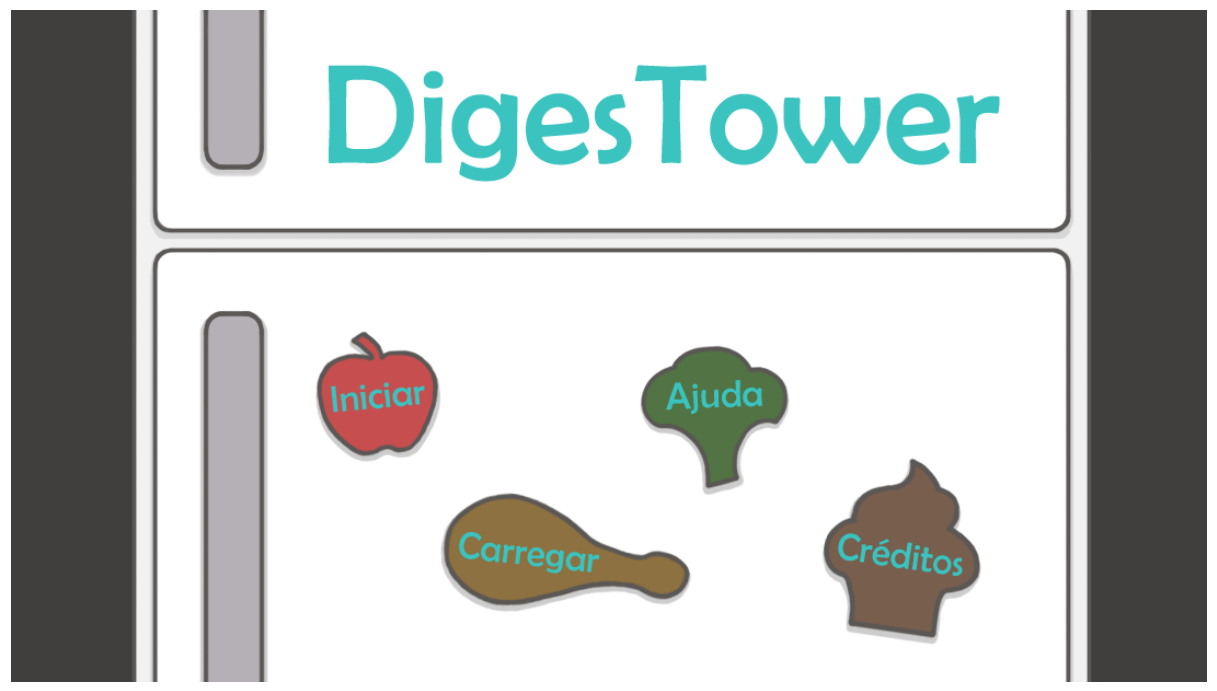

Figura 1: Tela inicial do DigesTower.

O jogo apresenta uma interface inicial na qual é possível selecionar um novo jogo ou carregar um jogo em andamento previamente salvo. Além disso, apresenta menus de ajuda em relação à mecânica do jogo e a consulta aos almanaques de informações do jogo como exemplo o de caracterização dos alimentos e das torres de defesa para maior compreensão e entendimento.

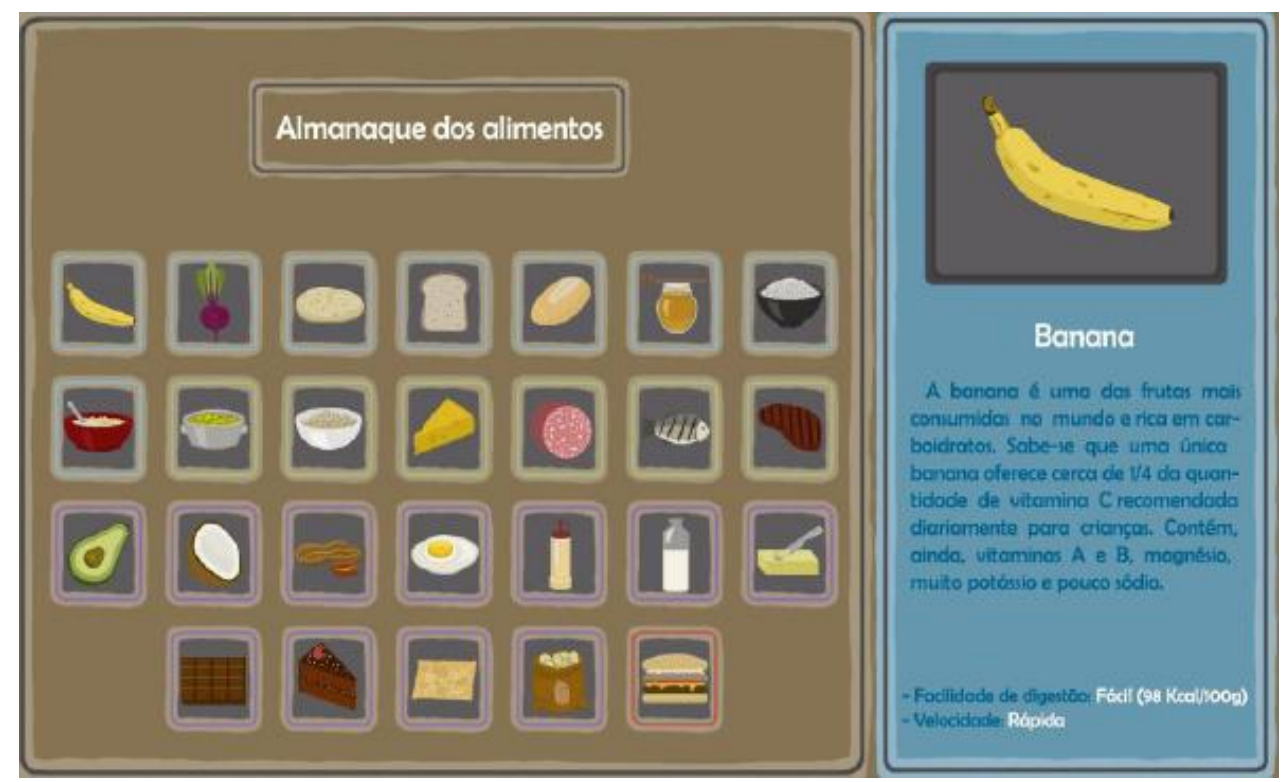

Figura 2: Almanaque dos alimentos.

O jogador poderá ainda consultar informações adicionais sobre os alimentos em um almanaque contendo as informações nutricionais (vitaminas, minerais, calorias fornecidas) e da dinâmica do jogo (velocidade do alimento e facilidade de digestão). 
V Congresso Brasileiro de Informática na Educação (CBIE 2016)

Anais dos Workshops do V Congresso Brasileiro de Informática na Educação (CBIE 2016)

O jogo também apresenta alimentos representativos dos principais grupos alimentares: carboidratos, proteínas e lipídios, bem como alimentos considerados "mistos", ou seja, que contenham mais de uma classe de alimentos. Para efetuar a digestão destes alimentos, o jogador deverá posicionar "torres" ao longo do trato gastrointestinal que representam as enzimas do corpo humano, responsáveis pela digestão destes grupos alimentares. As enzimas são diferenciadas por suas cores. As torres em azul representam as amilases, as roxas as lipases e as verdes as proteases.
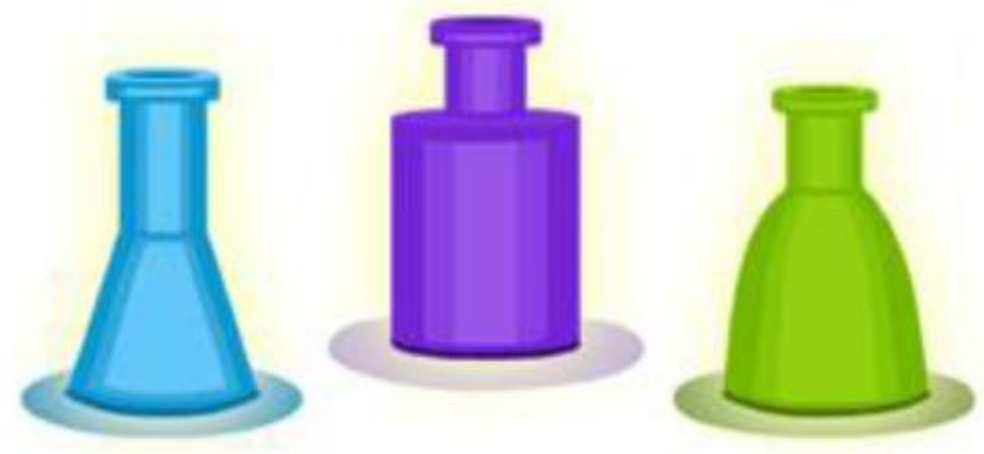

Figura 3: Exemplo das enzimas digestivas ("torres").

Na primeira fase (fase da boca) o jogador poderá selecionar apenas as torres de dente e de carboidratos, uma vez que as demais permanecem bloqueadas no menu até que o jogador avance para a fase seguinte. $\mathrm{Na}$ segunda fase (fase do estômago) são liberadas as torres que quebram proteínas, além das torres liberadas na primeira fase. Por fim, na terceira fase (fase do intestino delgado) são habilitadas as torres que quebram lipídios, sendo possível também, escolher todas as torres do jogo. Também são apresentadas informações sobre os grupos alimentares referentes à fase em que se encontra.

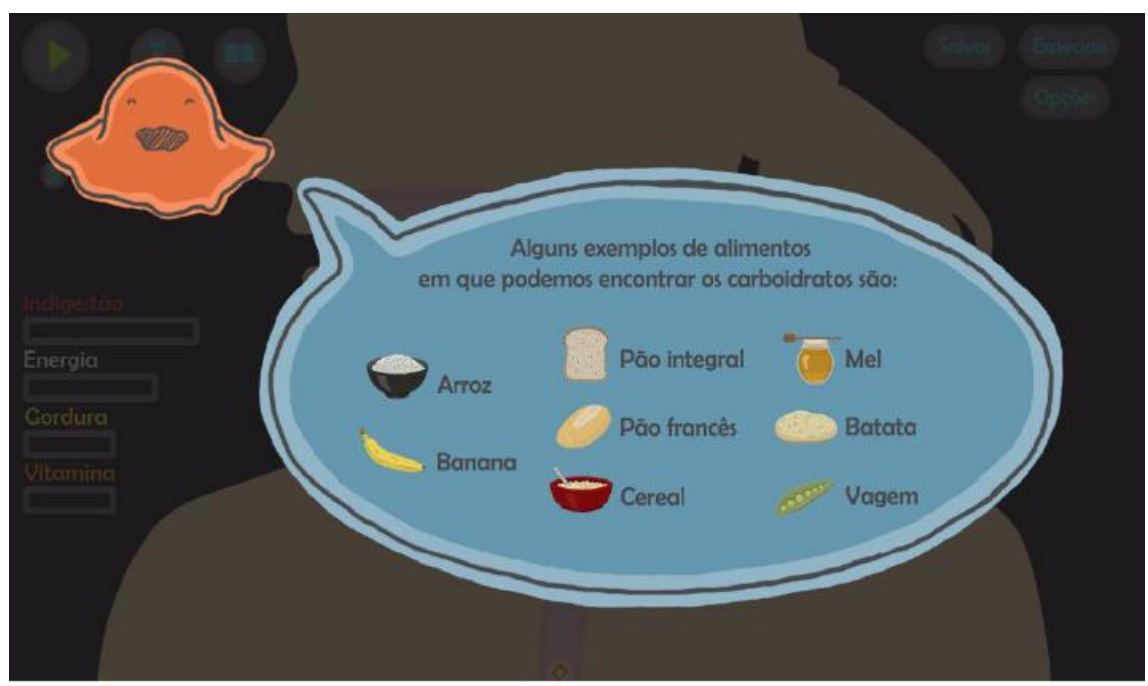

Figura 4: Exemplo de tela de informação 
V Congresso Brasileiro de Informática na Educação (CBIE 2016)

Anais dos Workshops do V Congresso Brasileiro de Informática na Educação (CBIE 2016)

Além disso, o mapa do jogo é desbloqueado gradativamente, iniciando com a boca e esôfago (primeira fase), depois estômago (segunda fase) e por fim o intestino delgado (terceira fase). Ao término o jogador deverá lidar com o trato gastrointestinal completo.

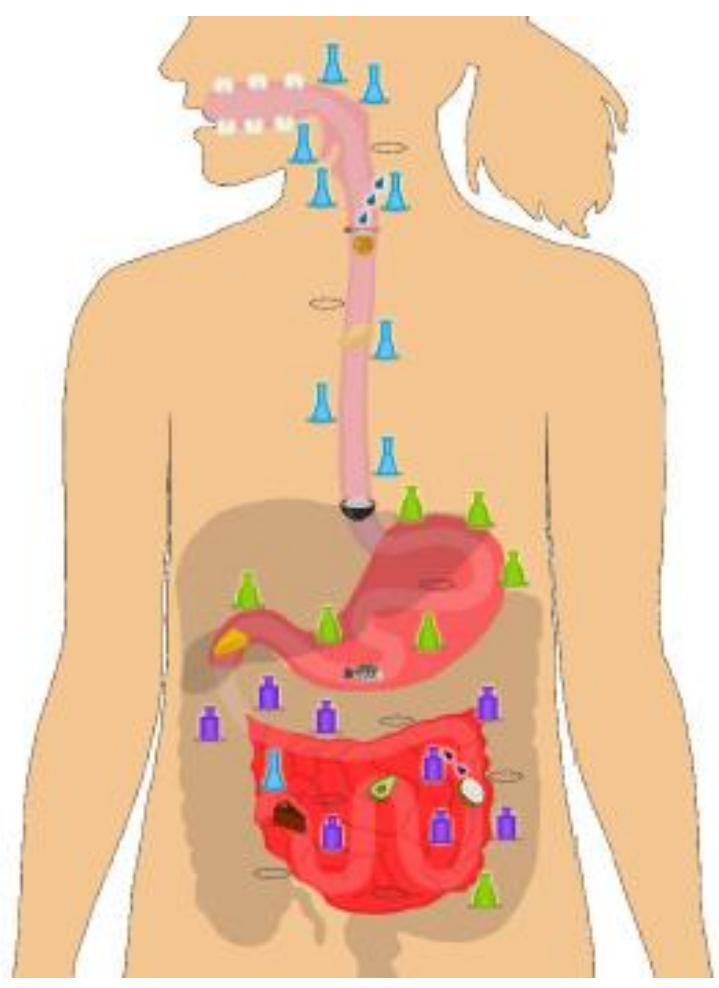

Figura 5: Mapa e mecânica do jogo.

Durante o jogo, o jogador pode escolher e evoluir suas torres (enzimas), escolher os locais adequados para cada torre, escolher os itens especiais e o melhor momento para execução de cada especial. O jogador vence ao realizar a digestão dos alimentos que são apresentados no decorrer do jogo ou perde se um grande número de alimentos passar ao longo do trato gastrointestinal sem ser digerido, ocorrendo assim uma indigestão.

\section{Considerações finais}

O uso de jogos e atividades lúdicas são os recursos preferidos pelas crianças, quando questionadas sobre como é que fariam para explicar alguma doença a outras crianças, caso pudessem ser elas os profissionais de saúde [Sparapani et al 2012]. Considerando estas informações, o serious game DigesTower foi proposto e desenvolvido visando suprir esta finalidade. Buscou-se oferecer um jogo de qualidade para que crianças escolares pudessem utilizá-lo como uma maneira divertida e atraente de aprender sobre a digestão humana, alimentação saudável e exercício físico, promovendo assim a saúde.

O jogo DigesTower se destaca por unir a temática de alimentação saudável à mecânica de jogos Tower Defense, com vistas a estimular o aprendizado do jogador e incentivar mudanças de comportamento. Houve uma grande preocupação em aliar os 
objetivos educacionais à jogabilidade, portanto o jogo contou com a participação de educadores e profissionais da área da saúde desde a concepção do mesmo até a sua implementação.

Ainda, um dos maiores diferenciais do jogo proposto é por se tratar de um recurso educacional gratuito e aberto. O DigesTower pode ser considerado um recurso didático tecnológico acessível a ser utilizado em escolas e unidades de saúde, além de poder ser reutilizado para a produção de outros jogos, por meio do acesso de seu código fonte.

Pode-se destacar que a identificação do jogador com o serious game através da imersão favorece o entretenimento e o desenvolvimento dos processos de ensino e de aprendizagem [Savi and Ulbricht 2008]. Nesse sentido, ao idealizar a concepção do jogo DigesTower buscou-se atentar a essas demandas. Durante o processo de elaboração buscou-se aliar as questões de aprendizagem com a interface, o áudio e a estética, de modo que fosse possível proporcionar maior imersão e adesão do jogador, a fim de atingir os objetivos pedagógicos propostos.

Posteriormente, através das avaliações dos especialistas foi possível verificar que o jogo tem grande potencial enquanto instrumento educacional, além de ter sido considerado uma estratégia diferenciada a fim de motivar a criança, auxiliar o profissional de saúde e contribuir para enfrentamento da obesidade infantil. O jogo foi avaliado positivamente pelos especialistas e considerado adequado para a aplicação futura com o público-alvo [Dias et al 2014].

A principal lacuna encontrada neste estudo foi a falta de oportunidade para testar o serious game com o público-alvo, o que seria de grande valia para uma maior efetivação dos resultados e possibilidade de validação do jogo na pratica enquanto intervenção. Entretanto, propõe-se uma continuidade de testes com as crianças, bem como a inserção do jogo em programas educativos de combate à obesidade. Assim, espera-se também buscar melhorar o jogo através do prosseguimento do mesmo em trabalhos futuros.

\section{Referências}

Baptista, M. I. M., \& Lima, R. (2006). Educação alimentar em meio escolar referencial para uma oferta alimentar saudável. Lisboa: Direcção Geral de Inovação e do Desenvolvimento Curricular. $35 \mathrm{p}$.

Bethke, E. (2003). Game development and production. Wordware Publishing, Inc.

Brasil. (2015). Caderno do gestor do PSE. Ministério da Saúde, Ministério da Educação. Brasília: Ministério da Saúde.

Dias, J. D., Mekaro, M. S., Lu, J. K. C., Sorrentino, G. S., Santiago, D. L., Otsuka, J. L., ... \& Zem-Mascarenhas, S. H. (2014). DIGESTOWER: jogo educacional para auxiliar o enfrentamento da obesidade infantil. In Nuevas Ideas en Informática Educativa - TISE 2014. Fortaleza (CE). 3009-3017.

Fu, F. L., Su, R. C., \& Yu, S. C. (2009). EGameFlow: A scale to measure learners' enjoyment of e-learning games. Computers \& Education, 52(1), 101-112.

Novak, J. (2011). Game development essentials: an introduction. Cengage Learning. 
V Congresso Brasileiro de Informática na Educação (CBIE 2016)

Anais dos Workshops do V Congresso Brasileiro de Informática na Educação (CBIE 2016)

Reis, C. E. G., Vasconcelos, I. A. L., \& Barros, J. F. D. N. (2011). Políticas públicas de nutrição para o controle da obesidade infantil. Rev Paul Pediatr,29(4), 625-33.

Savi, R., \& Ulbricht, V. R. (2008). Hipermídia Educacional, Jogos Digitais e Simuladores. In Conferência IADIS Ibero-Americana WWW/Internet Lisboa. Resumos da Conferência IADIS Ibero-Americana.

Schell, J. (2011). A Arte de Game Design: o livro original. Brasil: Elsevier.

Sparapani, V. C., Borges, A. L. V., de Oliveira Dantas, I. R., Pan, R., \& Nascimento, L. C. (2012). A criança com Diabetes Mellitus Tipo 1 e seus amigos: A influencia dessa interação no manejo da doença. Revista Latino-Americana de Enfermagem, 20(1), 117-125.

Sweetser, P., \& Wyeth, P. (2005). GameFlow: a model for evaluating player enjoyment in games. Computers in Entertainment (CIE), 3(3), 3-3.

Toscani, N. V., Santos, A. J. D. S., Silva, L. L. D. M. D., Tonial, C. T., Chazan, M., Wiebbelling, A. M. P., \& Mezzari, A. (2007). Desenvolvimento e análise de jogo educativo para crianças visando à prevenção de doenças parasitológicas. InterfaceComunicação, Saúde, Educação, 11(22), 281-294.

Tsuda, M., Sanches, V., Ferreira, T., Otsuka, J., \& Beder, D. (2014). Análise de métodos de avaliação de jogos educacionais. In: Proceedings of XIII SBGames 2014; XIII SBGames, 158-166.

Zechner, M., \& Green, R. (2012). Beginning Android Games (p. 158). Apress. 